\title{
Typical Landscape Tree Species Recognition Based on RedEdge-MX: Suitability Analysis of Two Texture Extraction Forms under Random Forest Supervision
}

\author{
Huaipeng Liu ${ }^{1 *}$, Xiaoyan $\mathrm{Su}^{1}$, Huijun $\mathrm{An}^{2}$ \\ ${ }^{1}$ School of Land and Tourism, Luoyang Normal University, Luoyang, Henan Province, 471934, China \\ ${ }^{2}$ College of Forestry, Inner Mongolia Agricultural University, Huhhot, Inner Mongolia, 010019, China
}

Received: 23 May 2021

Accepted: 30 August 2021

\begin{abstract}
The window size of texture feature extraction has a significant impact on the accuracy of tree species classification. The forms of all texture features share an optimal extraction window, and different types of texture features use their independent optimal extraction windows, which is conducive to tree species classification. In this study, we used a RedEdge-MX image as the data source and a random forest to determine two forms of the best texture extraction windows and construct their own best texture feature set. Then, we combined the best texture feature sets with spectral bands and the digital surface model (DSM) to analyze the difference between the two best texture extraction forms in tree species classification. The results show that the classification accuracy of the best texture feature set was significantly different between the two extraction forms. The overall accuracy of the first extraction form was $79.6365 \%$ and that of the second extraction form was $81.8915 \%$. When they are combined with a spectral band and the DSM, the classification accuracy of the latter was higher than that of the former (between $0.4295 \%$ and $2.2248 \%$ ). Hence, in the classification of tree species, the construction of the best texture feature set should be determined by the best extraction window for each feature type.
\end{abstract}

Keywords: RedEdge-MX image, tree species classification, texture extraction, suitability analysis, random forest

\section{Introduction}

The texture features of remote sensing images have important applications in tree species classification,

e-mail: gatestudy@163.com and the window size for texture feature extraction has a significant impact on the accuracy of tree species identification [1-3]. At present, the most common texture extraction method is to adopt uniformly changing windows for all types of texture features and then combine them with a specific classifier (e.g. maximum likelihood and random forest) $[4,5]$ until the classification accuracy of the tree species is no longer 
improved, and the current window is determined as the best window for texture feature extraction $[6,7]$. The extracted texture feature set corresponding to the best window is regarded as the best texture feature set for the tree species classification. After obtaining the best texture feature set of tree species classification based on the optimal window, the feature set is usually combined with other features of the image (e.g. spectral band, spectral index and hue, saturation and lightness colour space features), which can further improve the recognition accuracy of tree species $[8,9]$.

However, different types of texture features may have different optimal texture extraction windows. Therefore, when extracting multiple types of texture features to construct the best texture feature set for tree species classification, the same window is used to extract all types of texture features, and their independent window is used for extraction. Accordingly, the final constructed best texture feature set may be different. When the best texture feature set constructed by the two forms is used for tree species classification, the accuracy may be different. Furthermore, when they are combined with spectral bands, spectral index and digital surface model (DSM) for tree species classification, the accuracy may also be different.

The remote sensing data acquired by the RedEdgeMX sensor (made by Micasense company, USA) has five bands (i.e. blue, green, red, red edge and near infrared), and the spatial resolution of the acquired image can reach $0.1 \mathrm{~m}$ or even higher [10]. These data have an excellent spectral band and spatial resolution performance. In the identification of urban greening tree species, the performance of RedEdge-MX data is considered a problem that needs to be explored. To explore which texture feature set is most conducive to tree species identification using the same window or different windows for texture extraction, in this study, we utilised an airborne RedEdge-MX image acquired in Luoyang Normal University on January 3, 2020, as the data source. This study is based on the random forest classification of eight typical greening tree species that do not fall their leaves in winter: Ligustrum lucidum, Cedrus deodara, Photinia serrulata, Eriobotrya japonica, Magnolia grandiflora, Platycladus orientalis, Cinnamomum camphora and Trachycarpus fortunei. The results of this study are expected to provide a priori knowledge for the window selection of different types of texture feature extraction in tree species identification.

\section{Material and Methods}

\section{Data and Preprocessing}

The data used in this study were taken by the airborne RedEdge-MX sensor, which has five bands (blue, green, red, red edge and near infrared) and can obtain highspatial-resolution images. The detailed parameters of these data are shown in Table 1. The location of the imaged site is Luoyang Normal University in China. The imaging time was between 11:00 a.m. and 12:00 a.m. on January 3, 2020.

After the completion of data acquisition, we performed basic data preprocessing, such as image mosaic, band synthesis and image clipping. Then, we obtained an image covering the whole campus of Luoyang Normal University, with an area of approximately $2.03 \mathrm{~km}^{2}$. The false-colour display effect of the image (RGB532 combination) is shown in Fig. 1.

\section{Tree Species Investigation and Sample Collection}

We vertically cut the preprocessed image from the middle and obtained two parts. Then, we printed them on two sheets of $104 \times 60$ and $106 \times 60 \mathrm{~cm}$ papers. We utilised the paper images to investigate the main roads inside and outside of the school and the small forest in the school. We identified the trees corresponding to the paper image in the actual environment, circled the tree crowns in the paper image and recorded the tree species names. The typical tree species that do not fall their leaves during winter are L. lucidum, C. deodara, P. serrulata, E. japonica, M. grandiflora, $P$. orientalis, C. camphora and T. fortunei. Their photos are shown in Figs 2(a-h).

After the investigation, we marked the tree species on the electronic image in the form of a region of interest. Parts of them were used as training samples, and the other parts were used as precision validation samples (right side of Fig. 1). Detailed information on the surveyed tree species and pixel numbers of the training and validation samples are shown in Table 2.

Table 1. Band parameters of the airborne multispectral data.

\begin{tabular}{|c|c|c|c|c|}
\hline Band number & Band name & Spatial resolution $(\mathrm{cm})$ & Wavelength range $(\mu \mathrm{m})$ & Central wavelength \\
\hline 1 & Blue & \multirow{5}{*}{16.285} & $0.465-0485$ & 0.475 \\
\hline 2 & Green & & $0.550-0.570$ & 0.560 \\
\hline 3 & Red & & $0.663-0.673$ & 0.668 \\
\hline 4 & Red edge & & $0.712-0.722$ & 0.717 \\
\hline 5 & Near infrared & & $0.820-0.860$ & 0.840 \\
\hline
\end{tabular}




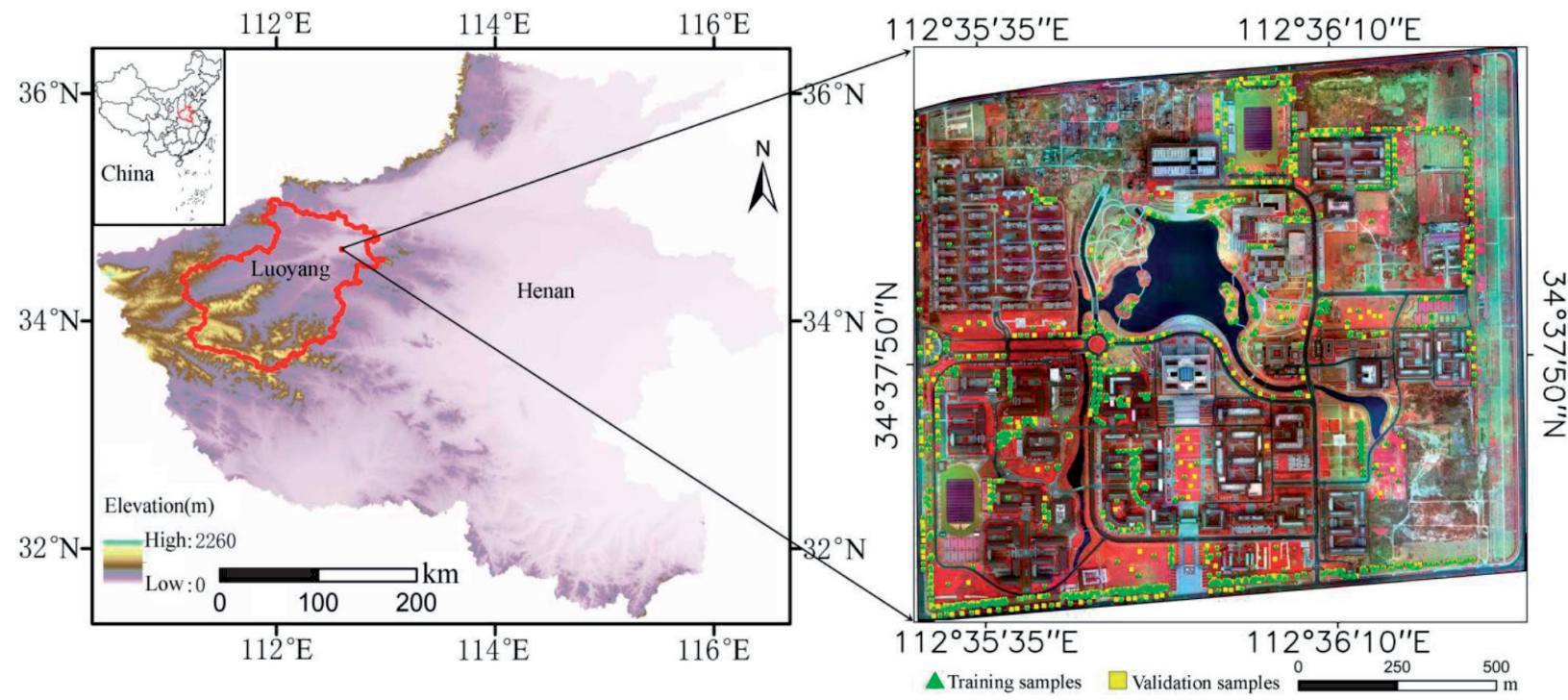

Fig. 1. Location of the study site in Luoyang Normal University, the RGB532 composition of the multispectral image and the distribution of the sample data.

\section{Extraction of Vegetations from the Images}

First, the DSM threshold of $[133,187.89]$ was used to initially mask the buildings and extract the building contour lines. Then, the lines were imported to MapGIS, and mismatched places were modified to make the vector lines more consistent with the building boundaries. Second, the updated building contour lines were used to build a mask, and the buildings were masked. Third, the generated mask file was used to mask the normalised difference vegetation index (NDVI) image, then edge-based segmentation was performed on the masked NDVI images (the best scale is 60.2), and the full Lambda schedule was used to merge (the best scale is 99) the segment patches. Finally, the object-oriented rule-based method was used, the threshold [0.11664, 0.63974] was set to extract the vegetation in the image, and the vegetation parts were retained. a)

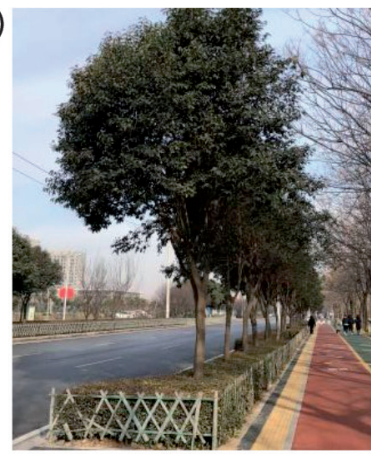

e)

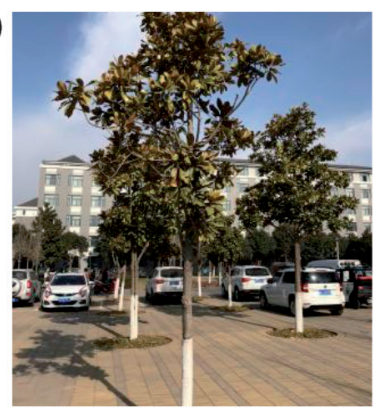

b)

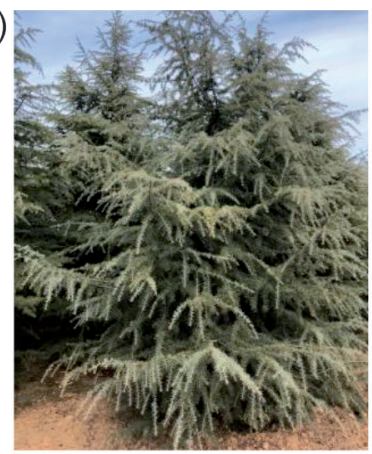

f)

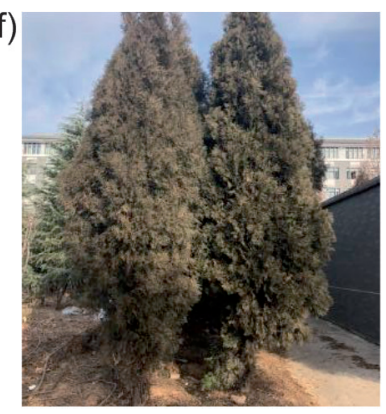

c)

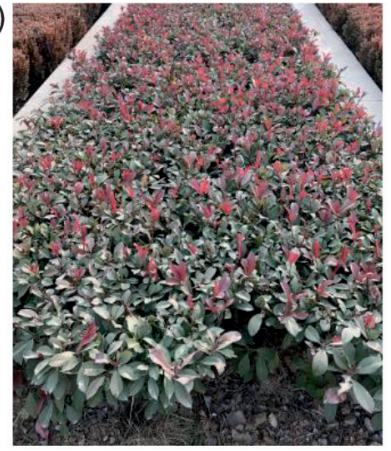

g)

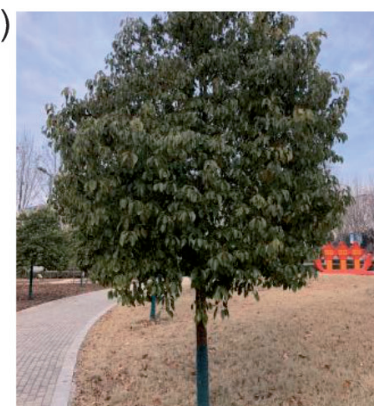

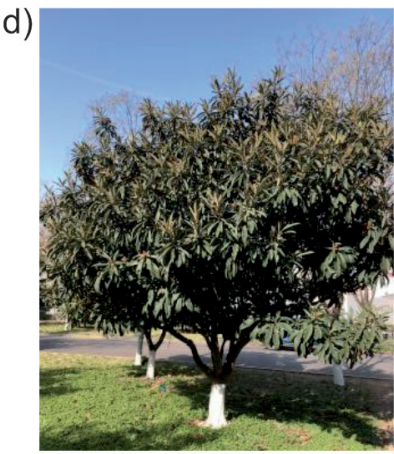

h)

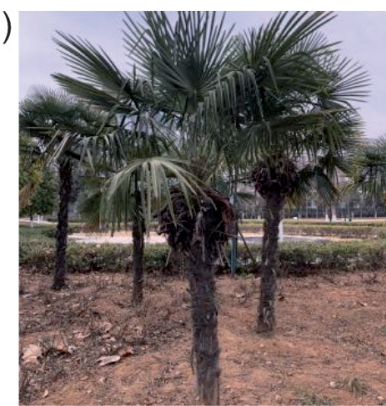

Fig. 2. Pictures of the eight landscape tree species, which were surveyed in winter. They were photographed on January $2,2021$. a) Ligustrum lucidum, b) Cedrus deodara, c) Ligustrum lucidum, d) Eriobotrya japonica, e) Magnolia grandiflora, f) Platycladus orientalis, g) Cinnamomum camphora, h) Trachycarpus fortune. 
Table 2. Surveyed tree species and their pixel samples.

\begin{tabular}{|c|c|c|c|}
\hline Latin names & Leaf type and phenology & Pixel numbers of the training samples & Precision validation pixels \\
\hline Ligustrum lucidum & Evergreen broad-leaf tree & 993 & 4491 \\
\hline Cedrus deodara & Evergreen conifer & 996 & 4591 \\
\hline Photinia serrulata & Evergreen broad-leaf tree & 971 & 4535 \\
\hline Eriobotrya japonica & Evergreen broad-leaf tree & 991 & 4589 \\
\hline Magnolia grandiflora & Evergreen broad-leaf tree & 922 & 4632 \\
\hline Platycladus orientalis & Evergreen conifer & 996 & 4522 \\
\hline Cinnamomum camphora & Evergreen broad-leaf tree & 994 & 3896 \\
\hline Trachycarpus fortunei & Evergreen broad-leaf tree & 978 & 4535 \\
\hline Grass & & 990 & \\
\hline
\end{tabular}

\section{Extraction of Image Texture Features}

Based on the co-occurrence measures of the ENVI 5.4 software, eight texture features were extracted for each band of the RedEdge-MX data: mean (MEA), variance (VAR), homogeneity (HOM), contrast (CON), dissimilarity (DIS), entropy (ENT), second moment (SM) and correlation (COR). In the texture feature extraction, we adopted two approaches. The first approach is to use the same texture extraction window to extract the eight texture features for each band (a total of 40 texture features are generated). In texture feature extraction, the processing window starts from $3 \times 3$ and sequentially increases until the overall accuracy of image classification does not increase (only rely on the extracted texture features for image classification). The second approach is to use the same window to extract the same type of texture features from all the five-band images. The texture extraction window starts from $3 \times 3$ and sequentially increases until the image classification accuracy is no longer increased. In this way, the best texture extraction windows for the eight kinds of texture features in tree species classification are obtained. By combining all kinds of texture features extracted from the best windows, we can obtain the optimal texture feature set for the tree species classification.

\section{Image Classification}

To determine the optimal texture extraction window of the two forms, we used a random forest [11] to classify high-dimensional texture features. After constructing the best texture feature sets of the images in two forms, we combined the spectral band and DSM with the two best texture feature sets, continued

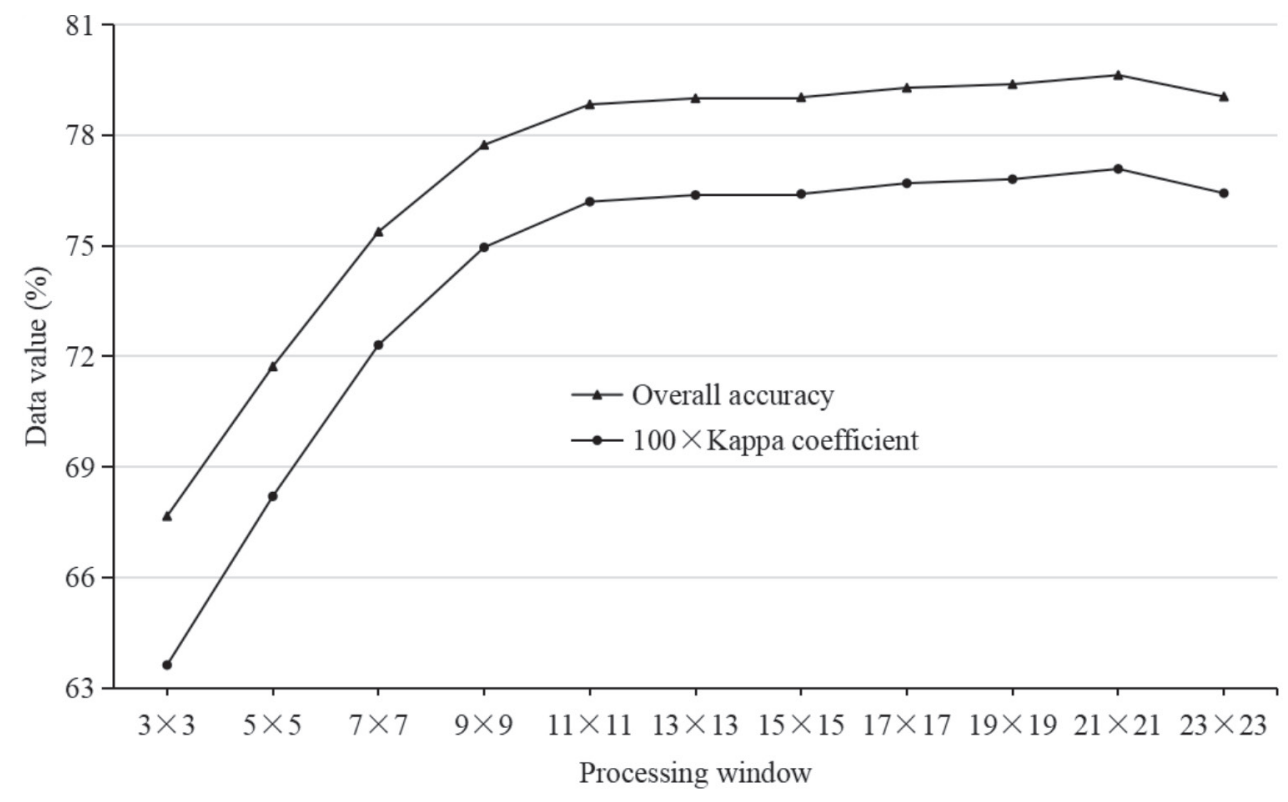

Fig. 3. Overall accuracy and $100 \times$ Kappa coefficient of the image classification under different texture extract windows. 


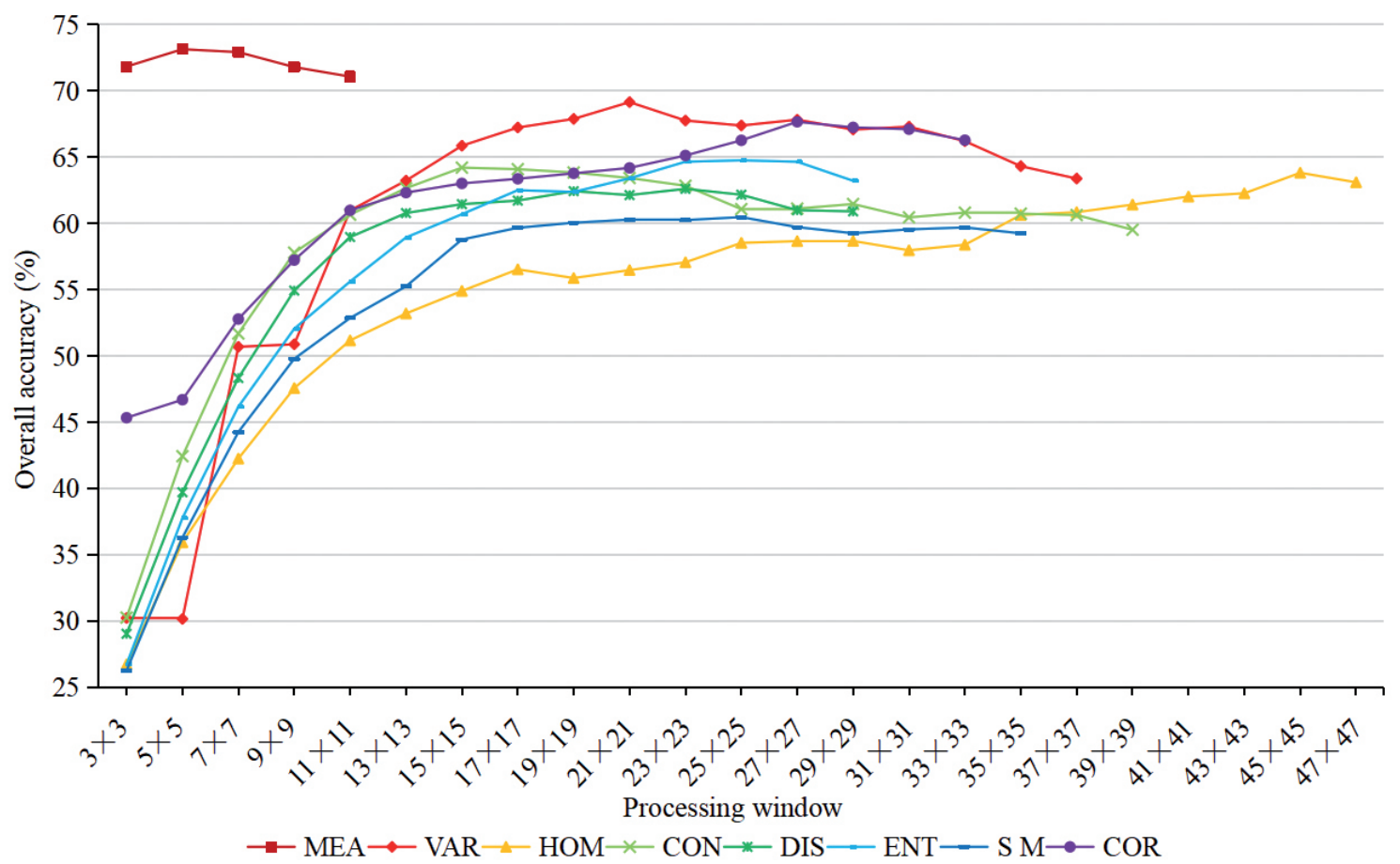

Fig. 4. Extraction of the same texture of five multispectral bands according to different windows and extraction of five textures as data sources to classify tree species using the random forest. The overall accuracy is counted in the corresponding processing window.

to use the random forest for classification, and compared the classification performance of the best texture feature set constructed by the two forms with the other feature sets.

\section{Result Evaluation}

After the image classification of all datasets, the verification sample was used for the accuracy test, and a confusion matrix was generated. The overall accuracy, kappa coefficient, producer accuracy and user accuracy were calculated by the confusion matrix and used for the quantitative evaluation. We used the comparison between the classification map and original image and the comparison between the classification maps to qualitatively evaluate the classification effect.

\section{Results and Discussion}

\section{Optimal Texture Extraction Window Analysis}

For the first texture extraction approach, the overall accuracy and 100× kappa coefficient were used to evaluate the best texture extract window required for the tree species classification. The experimental results are shown in Fig. 3.

As shown in Fig. 3, with the increase in the texture extraction window, the overall accuracy and kappa coefficient of the tree species classification are also increased. When the texture extraction window is $21 \times 21$, the two evaluation indexes obtain the maximum value, and when the texture extraction window is $23 \times 23$, the two evaluation indexes begin to decline,

Table 3. Performance of the obtained texture feature sets combined with other features in tree species classification under different texture extraction strategies.

\begin{tabular}{|c|c|c|c|c|}
\hline \multirow{2}{*}{ Data sets } & \multicolumn{2}{|c|}{$\begin{array}{c}40 \text { textures in the same window } \\
\text { (size is } 21 \times 21)\end{array}$} & $\begin{array}{c}\text { Combination of each type of texture in their } \\
\text { best window }\end{array}$ \\
\cline { 2 - 5 } & Overall accuracy\% & Kappa coefficient & Overall accuracy\% & Kappa coefficient \\
\hline 5 bands & 67.7733 & 0.6374 & -- & -- \\
\hline 40 textures & 79.6365 & 0.7709 & 81.8915 & 0.7963 \\
\hline 5 bands+ DSM & 75.9150 & 0.7290 & -- & - \\
\hline 40 textures+ DSM & 80.5085 & 0.7807 & 82.7333 & 0.8057 \\
\hline 40 textures+5 bands & 81.6855 & 0.7939 & 82.6563 & 0.8049 \\
\hline 40 textures+ DSM+ 5 bands & 82.6911 & 0.8053 & 83.1206 & 0.8101 \\
\hline
\end{tabular}




$$
\text { 言 }
$$$$
\text { 䍖 }
$$

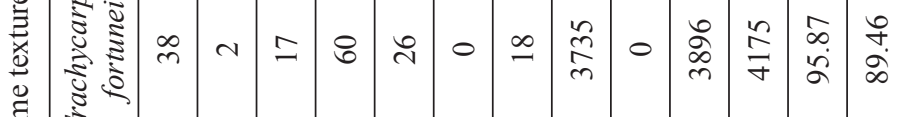

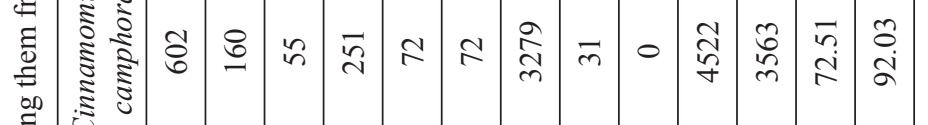




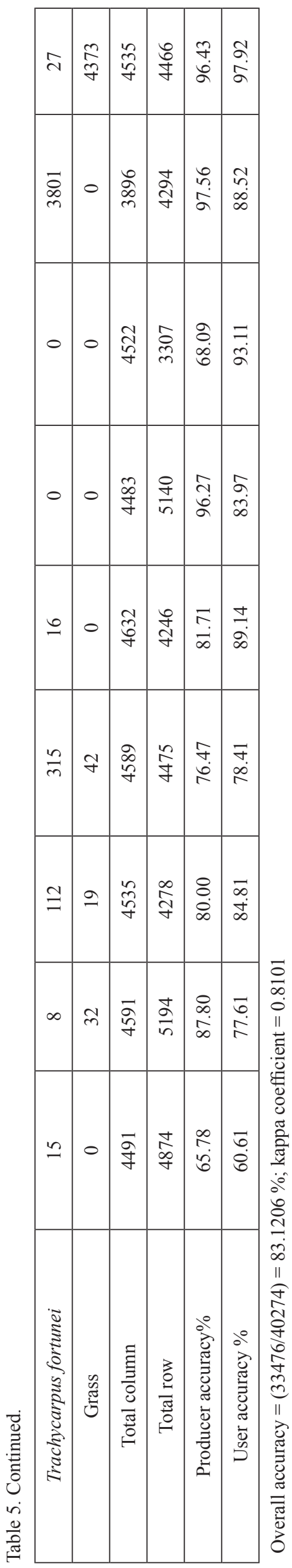

which indicates that the $21 \times 21$ window is the best texture extraction window in tree species classification in this study.

The overall accuracy of the tree species classification using the second form of texture feature extraction is shown in Fig. 4.

As shown in Fig. 4, in tree species classification, the best extraction windows for the eight types of texture features are mostly different. Some texture features require a smaller extraction window, such as MEA (the best extraction window is $5 \times 5$ ), and some texture features require a larger extraction window, such as HOM (the best extraction window is $45 \times 45$ ). The VAR, DIS, ENT, SM and COR have similar texture extraction windows (the best extraction window is between $21 \times 21$ and $27 \times 27$ ). Only the ENT and SM have the same texture extraction window (the best extraction window is $25 \times 25$ ).

The overall accuracy corresponding to the best texture extraction window required for the tree species classification is the highest in each texture feature type in tree species classification. Different types of texture features have different accuracies in tree species classification. The texture feature MEA achieved the highest accuracy (the overall accuracy is $73.1415 \%$ ), the texture feature SM achieved the lowest accuracy (the overall accuracy is $60.4782 \%$ ), and the classification accuracy of the other texture feature types ranges between those of the highest and lowest accuracies.

\section{Combined Texture Feature Set with Other Features}

Two texture feature sets (from extraction forms 1 and 2) were obtained under the two different texture extraction strategies. The accuracy of the tree species classification after combining it with the spectral bands and DSM is shown in Table 3.

Table 3 shows that regardless of whether the first or second form is used to construct the texture feature set, the accuracy (the overall accuracy is $79.6365 \%$ and $81.8915 \%$, respectively) of using the texture feature set to classify tree species is higher than that using the spectral band (overall accuracy is 67.7733\%) or the combination of the spectral bands and DSM (overall accuracy is $75.9150 \%$ ).

In Table 3, the overall accuracy of the best texture feature set extracted from the first form for tree species classification is $79.6365 \%$ (kappa coefficient is 0.7709 ), and the overall accuracy of the best texture feature set extracted from the second form for tree species classification is $81.8915 \%$ (kappa coefficient is 0.7963 ). The texture features extracted in the second form have a higher classification accuracy for tree species classification than those in the first form. Hence, the texture feature set constructed by the best extraction windows for different types of textures has a better effect on the tree species classification than the texture 
a)

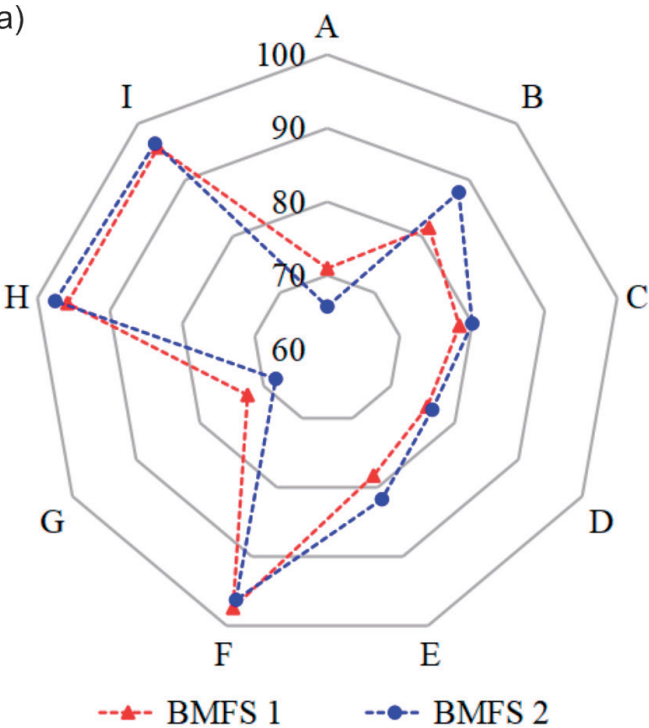

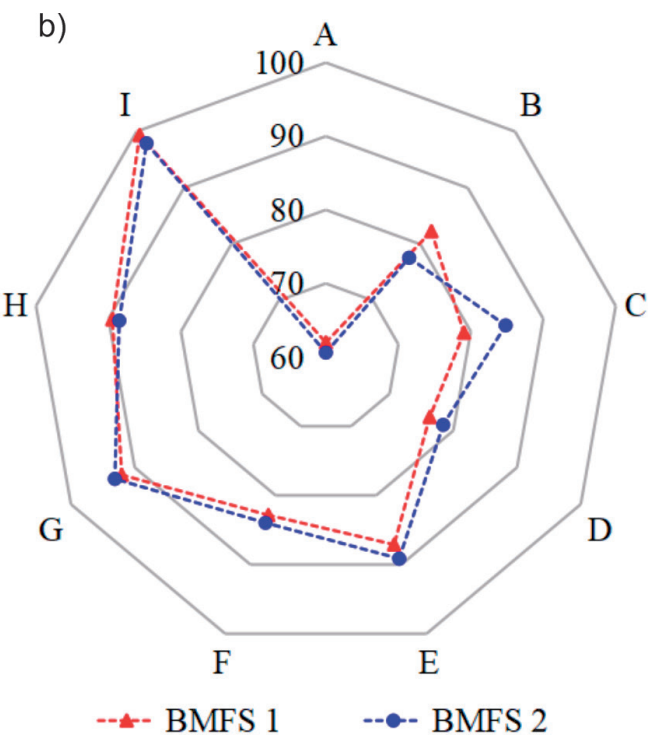

Fig. 5. Spider net diagram of the producer and user accuracies of the optimal feature combination under two texture extraction strategies. A, B, C, D, E, F, G, H, and I represent Ligustrum lucidum, Cedrus deodara, Photinia serrulata, Eriobotrya japonica, Magnolia grandiflora, Platycladus orientalis, Cinnamomum camphora, Trachycarpus fortunei and grass, respectively. a) Spider net diagram of the producer accuracy, b) Spider net diagram of the user accuracy.

feature set obtained using the same extraction window for all texture features.

The texture feature sets extracted in the two forms with the DSM and spectral bands and those extracted with the DSM and spectral band were combined at the same time. In the classification of tree species, the application effect of the texture feature set extracted and constructed by the second form is still better than that of the first one.

\section{Optimal Classification Result Analysis}

After the extraction of the optimal texture feature sets by the first and second forms, they were combined with the DSM and spectral bands in the tree species classification, respectively. The two confusion matrices with a good classification effect generated after the combination are shown in Tables 4 and 5, respectively.

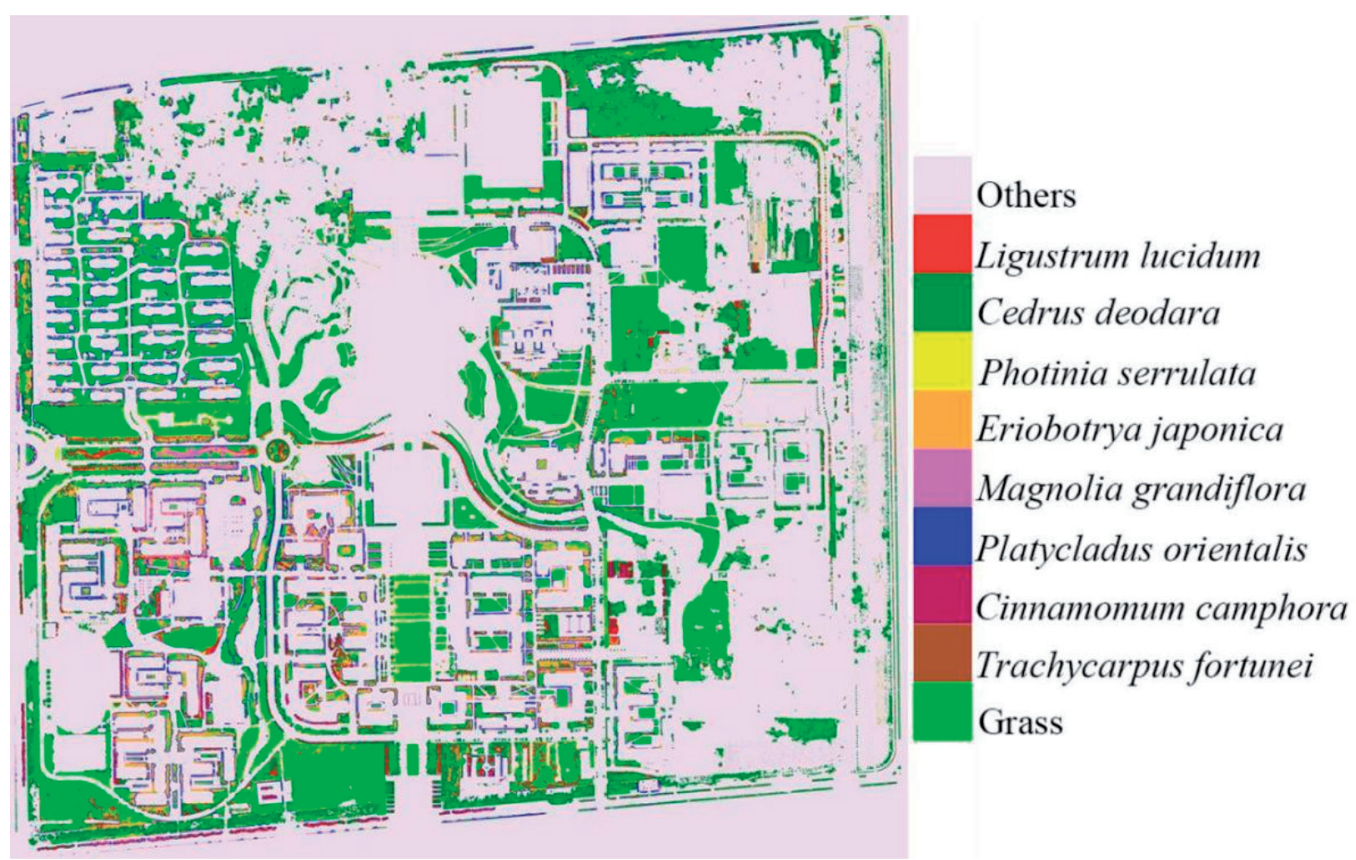

Fig. 6. Classification results of the highest overall accuracy. 
As shown in Table 4, the producer accuracy of the tree species recognition ranged from $70.96 \%$ (L. lucidum) to $97.43 \%$ (P. orientalis). Furthermore, the user accuracy of the tree species recognition ranged from 61.98\% (L. lucidum) to $92.03 \%$ (C. camphora). Large differences were observed in the producer and user accuracies among the various tree species. Meanwhile, large differences were also observed between the producer and user accuracies for the same tree species (e.g. L. lucidum, $M$. grandiflora, $P$. orientalis and C. camphora). Hence, the classification effect of the whole image is not particularly ideal.

As shown in Table 5, the producer accuracy of tree species recognition ranged from $65.78 \%$ (L. lucidum) to $97.56 \%$ (T. fortunei). Furthermore, the user accuracy of tree species recognition ranged from $60.61 \%$ ( $L$. lucidum) to $93.11 \%$ (C. camphora). Large differences were observed in the producer and user accuracies among the various tree species. Meanwhile, large differences were also observed between the producer and user accuracies for the same tree species (e.g. $C$. deodara, P. orientalis, C. camphora and T. fortunei). Hence, the combination of these groups of features and the classification effect of the whole image are not particularly ideal.

The producer and user accuracies obtained using the best mixed feature sets 1 and 2 (BMFS 1 and BMFS 2) are fitted to the spider net diagram, which are shown in Figs 5a) and b), respectively.

Fig. 5a) shows that the producer accuracies of the six plant categories in BMFS 2 are higher than those of BMFS 1, and those of the remaining three plant categories are lower than those of BMFS 1 . Fig. 5b) shows that the user accuracies of the five plant categories in BMFS 2 are higher than those of BMFS 1 , and those of the remaining four plant categories are lower than those of BMFS 1 . Hence, the classification effect of the best mixed feature set 2 is better than that of the best mixed feature set 1 . The findings show that different types of texture features are extracted according to their optimal extraction window, which is more suitable than the extraction according to a unified optimal window.

\section{Image of the Classification Results}

The landscape tree species classification results of the whole image under the optimal feature combination are shown in Fig. 6.

As shown in Fig. 6, the grassland area on the campus of Luoyang Normal University is relatively large and is well identified. The landscape tree species L. lucidum is mainly distributed on the side of the road, and it also has a good recognition effect. $P$. serrulata is distributed on the edge of the grass and is planted individually in blocks on the campus, which can also be well identified. In addition to the scattered distribution in the campus,
C. deodara is planted in a large area in the south of the campus and can be detected effectively. E. japonica, M. grandiflora, $P$. orientalis, $C$. camphora and T. fortunei are scattered on the campus and can also be effectively detected.

\section{Conclusions}

The most suitable for tree species classification among the best texture feature sets constructed by extracting all eight types of texture features from the airborne RedEdge-MX data were analysed according to the same best window and according to their respective best windows. In this study, after obtaining the best texture feature sets extracted in each form, they were combined with spectral bands and the DSM, and a random forest was used to classify tree species. The main results are presented as follows:

When all types of texture features are extracted using the same window, the optimal extraction window maintained a relatively centred window size $(21 \times 21)$. In each texture feature type extracted according to its independent window, the optimal extraction window of various textures is clearly different. Some texture features have a small optimal extraction window (such as the MEA, which is only $5 \times 5$ ). However, some texture features have a large optimal extraction window (such as the HOM, which is $45 \times 45$ ), whereas the others maintained a concentrated window size (between $15 \times 15$ and $27 \times 27$ ).

The best texture feature set obtained in the second form of tree species classification is higher $(81.8915 \%)$ than that obtained in the first form (79.6365\%). Hence, finding the best extraction window for each type of texture feature and combining the types of texture feature extracted by the best window are suitable in constructing texture feature sets for tree species classification.

When the two forms of best texture feature sets are combined with spectral bands and the DSM for tree species classification, the overall accuracy of the second form is also higher than the first form former. Our results further prove that the best texture feature sets constructed for tree species classification should be determined by the best extraction window of different texture types.

\section{Acknowledgements}

This work was supported by the Natural Science Foundation of Henan Province (Grant No. 202300410293) and the National Nature Science Foundation of China (Grant Nos. 32001250 and 42071198). We want to provide our gratitude to the editors and the anonymous reviewers. 


\section{Conflict of interest}

The authors declare no conflict of interest.

\section{References}

1. GONZÁLEZ-RUFINO E., CARRIÓN P., CERNADAS E.C., FERNÁNDEZ-DELGADO M., DOMÍNGUEZPETIT R. Exhaustive comparison of colour texture features and classification methods to discriminate cells categories in histological images of fish ovary. Pattern Recognition, 46 (9), 2391, 2013.

2. DIAN Y., LI Z., PANG Y. Spectral and texture features combined for forest tree species classification with airborne hyperspectral imagery. Journal of the Indian Society of Remote Sensing, 43 (1), 101, 2015.

3. DEUR M., GAPAROVI M., BALENOVI I. Tree species classification in mixed deciduous forests using very high spatial resolution satellite imagery and machine learning methods. Remote Sensing, 12 (23), 3926, 2020.

4. LIU H., AN H. Analysis of the importance of five new spectral indices from WorldView-2 in tree species classification. Journal of Spatial Science, 65 (3), 455, 2020.

5. LI D., KE Y., GONG H., LI X. Object-based urban tree species classification using bi-temporal WorldView-2 and WorldView-3 images. Remote Sensing, 7 (12), 16917, 2015.
6. ZHANG X.Y., FENG X.Z., JIANG H. Object-oriented method for urban vegetation mapping using IKONOS imagery. International Journal of Remote Sensing, 31 (1), 177, 2010

7. QUAN Y., ZHONG X., FENG W., DAUPHIN G., XING M. A novel feature extension method for the forest disaster monitoring using multispectral data. Remote Sensing, 12 (14), 2261, 2020.

8. PU R., LANDRY S. A comparative analysis of high spatial resolution IKONOS and WorldView-2 imagery for mapping urban tree species. Remote Sensing of Environment, 124, 516, 2012.

9. GHOSH A., JOSHI P.K. A comparison of selected classification algorithms for mapping bamboo patches in lower gangetic plains using very high resolution WorldView-2 imagery. International Journal of Applied Earth Observation \& Geoinformation, 26, 298, 2014.

10. AGARWAL A., KUMAR S. SINGH D. An adaptive technique to detect and remove shadow from drone data. Journal of the Indian Society of Remote Sensing, 49, 491, 2021.

11. VAN DER LINDEN S., RABE A., HELD M., JAKIMOW B., LEITÃO P.J., OKUJENI A., SCHWIEDER M., SUESS S., HOSTERT P. The EnMAP-Box - A Toolbox and application programming interface for EnMAP data processing. Remote Sensing, 7 (9), 11249, 2015. 